\title{
A Modelling Framework for Ambient Assisted Living Validation
}

\author{
Juan-Carlos Naranjo ${ }^{1}$, Carlos Fernandez ${ }^{1}$, Pilar Sala ${ }^{1}$, Michael Hellenschmidt ${ }^{2}$, \\ and Franco Mercalli ${ }^{3}$ \\ ${ }^{1}$ ITACA institute, Valencia, Spain \\ jcnaranjo@itaca.upv.es, carferll@itaca.upv.es, \\ msalaso@itaca.upv.es \\ ${ }^{2}$ Fraunhofer Institute for Computer Graphics Research IGD, Darmstadt, Germany \\ michael.hellenschmidteigd. fraunhofer.de \\ ${ }^{3}$ Centro di Cultura Scientifica Alessandro Volta, Como, Italy \\ franco.mercalli@centrovolta.it
}

\begin{abstract}
This paper describes a modeling framework that facilitates and streamlines the process of creation, design, construction and deployment of technological solutions in the context of AAL assuring that they are accessible and usable for senior citizens. The framework supports the design of the Human Interaction aspects of an AAL solution in all the stages of a user centered design methodology, putting in practice the guidelines for the verification and validation of the accessibility and usability facets. Two environments are defined: The authoring environment allow the definition of the user, environment and service models. The simulation environment is composed by software and hardware components that constitute a physical ensemble that in conjunction allow the ICT designer to implement actual Virtual Reality scenarios of AAL. It will be used to verify interaction designs and validate the accessibility of the AAL products by means of immersing the users in $3 \mathrm{D}$ virtual spaces.
\end{abstract}

Keywords: Modeling framework, AAL services, workflow technology, Ontology, Services choreography.

\section{Introduction}

Ambient Assisted Living (AAL) is the concept that embraces all the technological challenges in the context of Ambient Intelligence to face the problem of providing easy to use, accessible, affordable, sustainable and efficient solutions that improve the level of independence, promote the social relationships, leverage the immersion in the environments and encourage the psychological and physical state of the person. [1]

The user interaction in AAL services is perhaps one of the most complex interaction designs that a usability engineer can deal with. AAL refers to electronic environments that are sensitive and responsive to the presence of people and provide assistive propositions for maintaining and independent lifestyle, so here the challenge for the designer is to experiment with new and innovative modalities of interaction that must be, of course, accessible and usable. Some of the interaction modalities the 
designer can use in an AAL environment are speech recognition systems, gestural systems, context-sensitive systems, visual interaction, hearing interaction, tactile interaction, etc.

This paper describes a Modelling Framework to help the user-interaction designer to create immersive environments, user behaviour models, and to define new templates that permit the definition of new interaction modes and devices, using a formal specifications language and an immersive simulation platform to test the accessibility and usability aspects of AAL services. This modelling framework is used in the context of a User Centred design methodology and gives support to the different phases of such methodology.

\subsection{User Centered Design in the Modeling Framework}

To produce innovative and successful products it is needed to involve end-users, in our case Senior Citizens, throughout the whole development process. To do that we propose a methodology based on User Centred Design (UCD) that leads the AAL solutions development to a better position to ensure accessibility and acceptance of those services.

UCD is an approach that supports the entire development process with user-centred activities, in order to create applications which are easy to use and are of added value to the intended users. This approach is particularly useful when a new product or service is to be introduced, as it is the case of AAL solutions, because user-centred design draws together the practical, emotional and social aspects of people's experience bringing on the needed innovation that delivers real user benefit.

Despite the benefits, using UCD in the ICT industry is still confined because of cost and benefits. When systems become more complex, UCD evinces not only inefficient but also ineffective with respect to improving accessibility as there aren't available supporting tools. Designing and evaluating with manual approach is a timeconsuming endeavour, imposes a high work load on the designers and may lead to completion of the design search with premature, suboptimal solutions.

The modeling framework overcome these limitations by means of the definition of the AAL Solution User-Centered Design Methodology and the development of computer-aided supporting tools to be used by Interaction Designers and Usability Engineers at all development stages. The proposed methodology is defined taken as base the DIN EN ISO 13407[2], where a generalized human-centered design process for interactive systems is described.

The phases and the support that the Modeling Framework provides are:

- Concept phase. The main purpose of this phase is to elaborate the AAL Solution Requirements, including both functional and non-functional requirements. Usability engineer uses the simulation environment to present to seniors a wide range of real situations and elicit Senior reactions to identify Senior needs and goals. Interaction designer uses the authoring environment to model senior profiles (virtual seniors), interaction modes and interaction devices to visualize concept solutions. She/he also makes use of the simulation environment to explore concepts with Seniors 
- Design Phase. The main purpose of this phase is to define the AAL Solution specifications that will be used by the development team to implement the solution in the next phase. The conceptual design of the AAL solution addressing all aspects is created and reflected in low-fidelity prototypes, which are evaluated by Senior Citizens. The Interaction designer uses the authoring environment to design solution, creating virtual prototypes of the AAL service including virtual AAL-enabled spaces. Usability engineer uses the simulation environment to immerse Seniors in the AAL solution by means of the virtual prototype to experience with the new interaction devices, new interaction modes and elicit Senior reactions to identify early acceptance and accessibility issues

- Implementation phase. Main purpose of this phase is to build the AAL Solution Prototypes. The key design activity is transforming the validated conceptual design of the AAL solution into a concrete and fully detailed design including highfidelity prototypes that become really coded and fully functional. The Usability engineer uses the simulation environment to test the developed components against its accessibility features making use of the library of virtual seniors and providing improvements or corrective actions to the interaction designer. The Interaction designer uses the authoring environment to improve the design solution based on the input received from the usability engineer.

- Test phase. The main purpose of this phase is to validate the final implementation of AAL Solution Prototypes to ensure it satisfies business, market and user requirements. The usability engineer uses the simulation environment to test final implementation, first detecting usability issues with the automated tool and then with real Seniors

\subsection{The Modeling Framework Environments}

The modeling framework address the problem of designing user interfaces and interaction modes which are accessible and accepted by elderly people living in Ambient Assisted Living environments.

The modeling framework is a tool that can be used across all the stages of the design cycle. According to the workflows of a User-Centered design methodology, the framework set up two work environments:

- Authoring environment

- Simulation environment

The core of the authoring environment is the authoring tool. This tool allows the creation of 3 different models:

- AAL Service Interaction model (any present or future AAL service)

- Environment model (smart house, airport, station, work-place,...)

- User Behavior Model (mobility impaired, visual impaired, ...)

These models will be designed using a library of templates that can be shared and enriched among the interaction engineers. The specification of the templates is made by means of ontologies.

The models created in the Authoring environment are used in the Simulation environment to: 


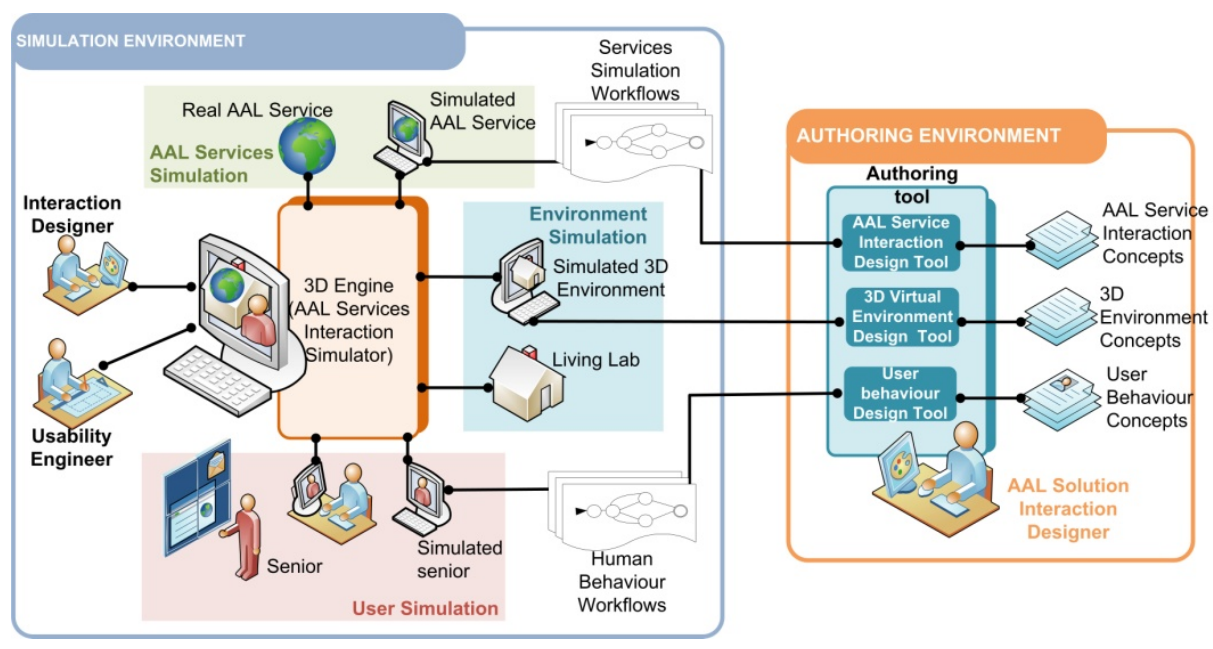

Fig. 1. Modelling Famework environments

- Validate the concepts of the interaction

- Produce the detailed design of the interaction

- Support the building of the interaction

- Validate the interaction

\section{The Modeling Framework tools}

The authoring and the simulation environments provide to the interaction designer with a set of tools to fully understand the usability and accessibility facets of the AAL solution. These tools are detailed below.

The environment model builder is the tool for creating not only the $3 \mathrm{D}$ scene of the environment but also the individual behavior of the elements deployed on it. This editing tool imports the 3D scene from any graphical user interface (so-called 3D designer tools, e.g 3D studio Max) and assigns the elements that are relevant for the interaction with the AAL service with the desired behavior.

The user model characterization tool is the tool devoted to simulate the user side interaction. With this tool the designer can simulate how and when the interaction will take place. In this way the designer can simulate several user behaviors and store them in the model library. The idea behind that is to create a collection of different user behaviors to test the service without the exhaustive presence of the real user in the framework.

The AAL service compositor is the designer tool for creating the workflow of the AAL service. It receives as input the environment model and the user model and permits the designer the establishment of the links between the different elements of the scene. The objective is to build the functionality and behavior of the desired real service.

The model library is the repository of all the models developed with the tools of the authoring environment. It works at as structured and well organized set of models 
where the designed can share, search and contribute to the enrichment of the framework in a collaborative way.

The simulation control panel is the core of the simulation session. It is the configuration tool of the simulation and allows the tuning of the parameters for the simulation, the launch and stop of the simulation and, what is more important, the final design of the simulation. This point should be explained in more detail; one of the key features of the framework is that the designer can play with different configurations regarding the actors, the environment and the services. He can create a simulation with the user model or the real user, with the environment model or with real part of it and with a complete service model or with a partially implemented real service.

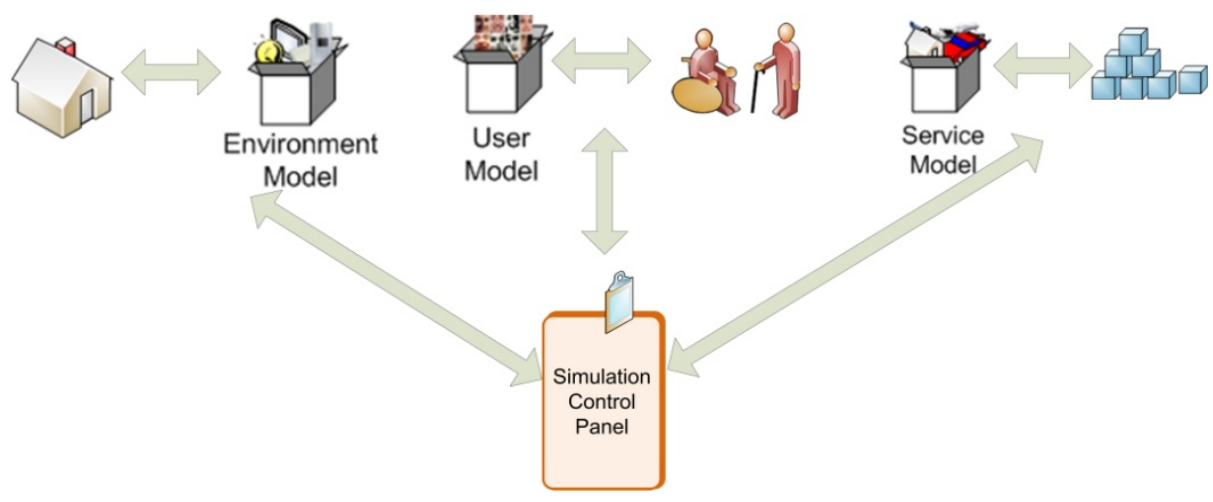

Fig. 2. Models and Simulation control panel

This strategy allows the swapping between a model of a device and the real device if it is available or the swapping between a part of the service model and a developed functionality of the service, maintaining the flexibility of the environment and supporting the User centered design process.

The 3D simulation browser is the render engine for the 3D scenes. The user is immersed on it and then is allowed to interact with the environment and the representation of the service that runs on it. It is based in the development of the Fraunhofer Institute for Computer Graphics Research IGD and is called InstantReality [3] framework which is a high-performance Mixed Reality (MR) system.

The interaction between the InstantReality player and the user is done by means of the 3D interaction devices. The 3D interactions devices are the physical interfaces that the user uses to "touch" the objects in the InstantReality, some example of devices being used are gloves, pointers, wiimote, etc.

The lab verifier is the next step in the immersion of the user in the simulation environment. Instead of using the 3D browser, the user is invited to use the service in a real environment, using real interactions devices, normally in the last phase of the refinement. Here the lab is integrated with the service model to evaluate the final aspects of usability of the solution. The lab verifier is the last step in the simulation, but the same infrastructure is used to validate individual interaction devices. In this case the real devices are integrated with the $3 \mathrm{D}$ environment and with the service model and other device models as well. 


\section{Results}

Different technologies have been used to create the framework. In any model there are two views that should be defined: The static view and the dynamic view. The first one describe the structure and the inner relationships of the model, the second describes how the different parts of the model interacts each other as time goes. By the other hand, the framework requirements imply that the models must be fully executable.

\subsection{The Static Model: Ontologies}

There is not a unique way to model a domain. As an abstract description of a certain field of knowledge, several approaches are possible, like in the Entity-relationship model, in which several solutions can solve the same problem.

Managing such amount of information requires a robust an efficient solution. Ontologies can solve that because they were conceived to define concepts and their relationships with other concepts in a hierarchically way in order to share the knowledge about a certain domain with the aim of reusing it and make feasible the analysis of the knowledge about the domain. Together with task ontologies, domain ontologies complete the specification of application ontologies. They also provide other features like reasoning that allows classifying, inferring or deducing new entries as well as provide the right answers when querying.

In order to prevent creating an ontology from scratch, and not reinventing the wheel again, the ontology languages (as OWL is) include statements that allow importing and reusing other existent ontologies (in OWL is owl:imports), with the aid of this kind of construction the ontologies can grown easily the level of detail provided.

This is the approach adopted in this framework. Although there are other languages or solutions (KIF, Frame based, RDF, etc.) to create and maintain an ontology, the decision was to choose OWL as the language to develop the ontologies managed by the project. This decision was taken because there are a lot of tools that allows the development, the access and reasoning of the model and instances hold by the ontologies, as well as OWL is a W3C Recommendation since 2004[4]

When talking about the environmental domain, the most frequent environments which the AAL services target are those places which are "enclosed" spaces, such as a residence, a hospital, a house, etc. In most of the cases these services are deployed in a house, at the user's home. There are other AAL services that were thought for more "open" spaces like a train station, a metro station, etc. Each type of environment has its own objects; some of them are proactive, whereas others have a reaction when a stimulus arrives, i.e., they have a response to the environment.

In order to model these kinds of environments, the concepts related to spaces themselves have to be identified, i.e., rooms, walls, etc. Furthermore, the different objects placed inside these spaces have to be specified, including furniture, appliances, sensors, and actuators. Objects have a set of properties, such as their type and their dimensions; their dimensions can be taken into account in order to compute the free space between objects, as well as to consider accessibility issues. In addition to the types of objects that can be found in the environment, there are other features of the environment that must be also taken into account when modeling it like the amount of light, timing (intervals, time in the day), season of the year, etc. 
The user model refers to the model of those who will be using AAL products and services. In this case, the user is mainly the person who uses, maintains or is affected by the use of the system under consideration, that is, the elderly people. When modeling the elderly people some considerations should be taken, there are normative models of changes on various dimensions, and a large body of knowledge about how age-related chances appear typically and on average in an aging population. These changes can roughly be divided into psychological, social, and physiological/ biological. Psychological aging contains several factors, such as cognitive functioning, psychomotor performance, and personality. Social changes related to aging can be viewed on both the individual and the interpersonal level. [5][6].At the individual level, areas subjected to change are such as personal roles and attitudes With increasing age, social roles usually change or are in transition; e.g. from parent to grandparent, from employee to retiree, and from married person to a widower. As the personal roles change, the relationship to the surroundings changes too, and accordingly, attitudes and values may change. Age-related biological and physical changes include bodily changes, e.g. changes in blood circulation systems, sensory systems, immune system, body mass, and muscles. Many of these changes affect the individual's capacity to function, and some of them predispose the individual to illnesses.[7]

When modeling the service we have to focus in the interaction of the user with such service. The Ambient Intelligence vision in which the AAL services are enclosed promotes the use of the implicit Human Computer Interaction defined by Schmidt, 2005 [8]. The implicit human computer interaction (iHCI) takes the users context into account when creating new user interfaces for ambient intelligence. The basic idea of implicit input is that the system can perceive the users interaction with the physical environment and also the overall situation in which an action takes place. When modeling iHCI the focus goes from the user dialog to the capture of the user context, for example, the location of the user, the movement of the user and the activities he or she are carrying out.

\subsection{The Dynamic Modeling: The Workflow Technology}

In order to describe the interaction in terms of execution, the framework will combine the use of those ontologies with the use of workflows. In the project, the Workflow Technology is used to deal with the problem of dynamic execution of processes choreography. Workflows are formal specification of processes execution which can be dynamically executed using a software piece called workflow engine.[9] Workflows can be defined to specify the behavior of the user and the environment in the interaction simulation framework. There are a lot of workflow engines and workflow formal languages available in the market which could be used, like JBPM, Windows workflow Foundation, Staffware, COSA, FLOWer ... etc. In the classic literature there are a lot of solutions to model the behavior of an entity whatever it is: an actor, a system, a service, etc. There are solutions based on rules that try to model the behavior by using statements composed by two parts: the precondition "IF" + the action "THEN". One of these kinds of rules is the called Event-Condition-Action rule. These rules were thought to act in response to an event launched by the system, but they had limitations to cover all the possible cases that are needed to model an entire behavior. Little by little the structure of the rules was changing in order to model the flow 
according with the real behavior of the entity to be modeled. At this point other type of solutions appeared based on modeling the behavior by using workflows, which can be defined using rules or not and also can use rules within them or not. Currently a workflow is defined as the formal specification of a process. It defines actions, performed by humans or by computerized systems, and the set of allowed transitions among them that define the possible paths that a custom process can follow.

The languages used to specify workflows can be either based on theoretic models (like Petri nets or parallel automaton) or based on models oriented to the representation (like Business Process Modeling Notation), the UML 2.0 Activity Diagrams or the XML Process Definition Language, and they are executed in different workflow interpretation systems, which use an executable specification of the workflow. Regarding to the executable languages, the Business Process Execution Language (BPEL) [10] is a language based on XML. To perform the execution of the workflows it's need a specialized workflow engine. After some considerations it was chosen as the most adequate the JBPM[11], a workflow management system that supports BPEL.

\subsection{The Choreographer}

The 3D browser and the workflow engine work together by means of the process choreographer. Choreography is defined by OMG as "the specification of interactions between autonomous processes" [12]. Choreography is done at a global level, and all (or most) of the participants (in the process) are equally involved in, the choreography has an objective point of view of the process. The important for the choreography is the protocol; it describes interactions from a global perspective, the set of messages managed during all the process, which are exchanged between the participants without the aid of any controller. Choreography is collaborative; each participant describes the part they play in the interaction [13].

This is the approach followed by the framework. It was intentionally chosen because we want to preserve the independence of the different processes. The role of the

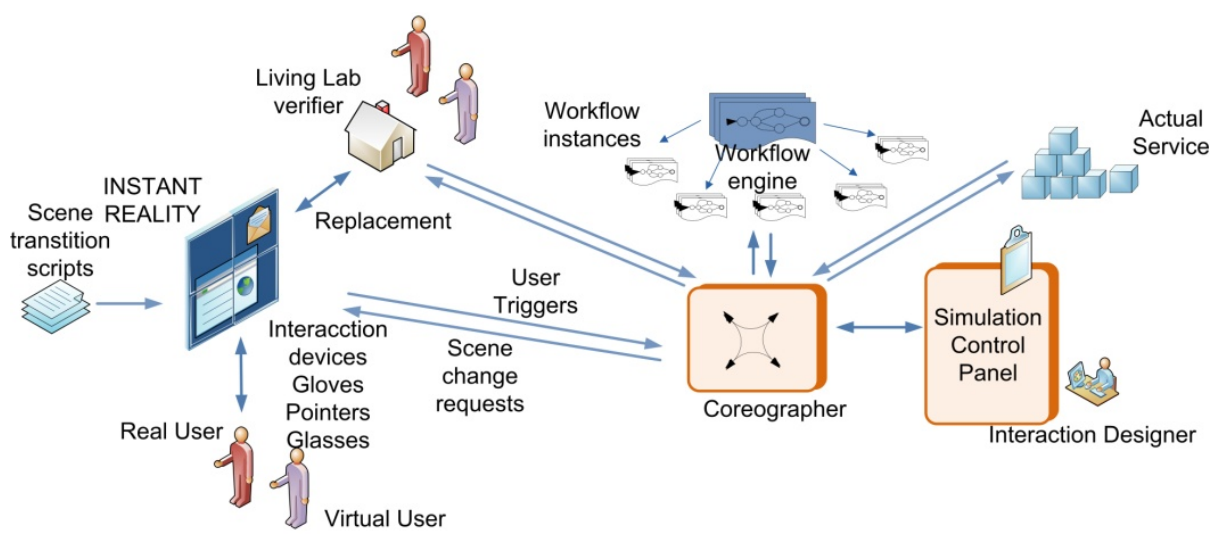

Fig. 3. Choreographer 
choreographer is to allow the communications of the events that should be spread among the different workflow instances of the models, the events that are transformed to signals going to the $3 \mathrm{D}$ browser and the events that the user triggers through the $3 \mathrm{D}$ scene and go to the workflows of the service model and the environment models.

\section{Conclusions and Future Work}

The paper describe a modeling framework for validate the usability and accessibility of AAL services inside of a User Centered Design methodology. The framework has been designed to model the interactions of a service, the environment where it will be delivered and the user who will use them. The framework provides to the designer with tools to create the models, to share them and to enrich them and gives the flexibility to control the simulation session and swap between the models and the reality.

With these tools in hands of the designer, the ICT AAL system designers will be able to create fully featured AAL ambient with sensors and interfaces. In concordance with the User Centered Methodologies, during user-designer interview sessions and small focus group sessions, selected Senior Users will be to immerse in virtual experiences to elicit the most optimal UI that maximize accessibility to the given services. This virtuous circle might be repeated all along the process of designconstruction-testing process, providing tremendous support and assistance to ICT designers to fine tune their developments of UIs solutions.

Not all the work is in the scope of the project. There is a long way to cover all the designer's requirements, especially in the modeling of user characteristics. The research field that will lead to a complete model of a virtual user is still far to be achieved. Other area of improvement and research will be a more immersive virtual reality experience. The 3D platforms and the interaction devices to be used with them can be improved with better and non intrusive systems.

Acknowledgments. This work has been partially funded by the European Union in the context of the VAALID project. Our gratitude to the VAALID consortium composed of the following partners: Siemens S.A Fh-IGD, UniPR, VOLTA, UPM, UID, SPIRIT and ITACA..

\section{References}

1. Steg, H., Strese, H., Hull, J., Schmidt, S.: Europe is facing a demographic challenge Ambient Assisted Living offers solutions (2005)

2. International Organization for Standardization. DIN EN ISO 13407

3. http://www. instantreality.org

4. W3C World Wide Web Consortium, http: / /www.w3 . org/TR/owl-guide/

5. Cumming, E., Henry, W.: Growing old: the process of disengagement. Basic Books, New York (1961)

6. Atchley, R.: The social forces in later life: An introduction to social gerontology. Wadsworth Publishing Company, Belmont (1971) 
7. Gogging, N., Stelmach, G.: Age-related deficits in cognitive-motor skills. In: Lovelace (ed.) Aging and cognition. Mental processes, self-awareness and interventions. Elsevier Science Publishers, Amsterdam (1990)

8. Schmidt, A.: Interactive Context-Aware Systems Interacting with Ambient Intelligence. In: Ambient Intelligence, pp. 159-178. IOS Press, Amsterdam (2005)

9. Workflow Management Coalition, http : / /www . wfmc . org

10. OASIS Web Services Business Process Execution Language (WSBPEL), http: / /www . oasis-open.org/committees/wsbpel/

11. http: / /www.jbpm. org

12. The Object Management Group (OMG), http: / / www . omg . org

13. Peltz, C.: Web Services Orchestration and Choreography. Hewlett-Packard Company. IEEE Computer Society Press (2003) 